\title{
GESTIÓN TERRITORIAL MEDIANTE ESTRATEGIA DE ASOCIATIVIDAD PARA CASANARE - COLOMBIA
}

\section{TERRITORIAL MANAGEMENT THROUGH ASOCIATIVITY STRATEGY OF CASANARE COLOMBIA}

\author{
Adriana Posada Arrubla ${ }^{1}$, Antonio Esteban Campuzano ${ }^{2}$, Ana Elena Berrocal Mendoza ${ }^{3}$ \\ ${ }^{1}$ Economista Agrícola, Esp. Gestión Social y Ambiental, M.Sc. Planeación Urbana y Regional, Docente Facultad de Inge- \\ nierías. U.D.C.A, Calle 222 No. 55-37, Bogotá D.C., Colombia, e-mail: aposada@udca.edu.co; ${ }^{2}$ Ingeniero Geógrafo y Am- \\ biental U.D.C.A, Joven líder de Casanare. Transversal 11 No. 1a-08, Hato Corozal - Casanare, Colombia, e-mail: aesteban- \\ campuzano@hotmail.com; ${ }^{3}$ Ingeniera Ambiental, Esp. Gestión Ambiental, M.Sc. Políticas y Desarrollo Sostenible, Institut \\ Catholique de Paris-Francia, e-mail: anaberrocal19@hotmail.com
}

Rev. U.D.C.A. Act. \& Div. Cient. 20(2): 445-456, Julio-Diciembre, 2017

\section{RESUMEN}

Ante la necesidad de alternativas de desarrollo en el territorio, la integración entre vecinos se hace cada vez más necesaria y posibilita compartir, no solo responsabilidades sino potencialidades, especialmente, a nivel local. En el caso del departamento de Casanare, en Colombia, se aplicó un método de análisis geográfico-ambiental, para motivar la gestión de una estrategia territorial entre entes municipales. En este estudio, se logró generar cartografía síntesis de los subsistemas ambiental y socieconómico, cruzados con entrevistas a la comunidad, para el planteamiento de una estrategia de gestión, a partir de la asociatividad de tipo funcional, entre seis municipios.

Palabras clave: Desarrollo territorial, articulación supramunicipal, estrategia territorial.

\section{SUMMARY}

The need for alternative development in the territory, the integration between neighbors becomes increasingly necessary and makes it possible to share not only responsibilities, but potential, especially at the local level. In the case of the Department of Casanare in Colombia, a thesis analysis method was applied to motivate the management of a strategy of territorial between municipal entities. In this study were managed to generate mapping environmental synthesis of subsystems and socio-economic, with interviews with the community, to the approach of a management strategy, from the associativity of functional type, between six municipalities.
Key words: Territorial development, joint supra-municipal, territorial strategy.

\section{INTRODUCCIÓN}

La gestión territorial, entendida como la sumatoria de acciones colectivas para hacer realidad los sueños, que llevan a lograr una armonía en la relación sociedad-naturaleza, exige intervención puntual en un espacio determinado, mediada por estrategias, que permitan el logro gradual de ese sueño colectivo, de tal manera que, a su vez, la sumatoria de estrategias, generan una fuerza y un tejido de interacciones compartidas, capaz de dinamizar un futuro más prometedor y de hacer proyectos innovadores y particulares.

Por ello, es importante indagar por las formas en que se puede establecer la estrategia colectiva, que permita a los habitantes de un territorio crear un mejor futuro. Según Cabeza (2015) es necesario reorientar las políticas y los procesos de gestión territorial, para alcanzar niveles de integración, impulsada por proyectos de desarrollo colectivos. Para Rojas (2016), los objetivos de la gestión territorial deben ser medidos por el logro de bienestar para toda la población y la adaptación armoniosa al medio, sin sobrepasar los límites ecológicos. De hecho, el territorio ya está articulado de manera natural, lo que hace falta es hacer de la división política-administrativa, una oportunidad de acción en conjunto; lo que implica integración de intereses. Y aunque en cada localidad o municipio existe diversidad de intereses colectivos, indefectiblemente, la naturaleza permite mitigar estas diferencias y aunar esfuerzos, para un mejor futuro común (Posada, 2014). En suma, estas acciones o proyectos locales no pueden responder a una lógica inmediatista, sino a una 
gradualidad de largo plazo, enlazada con el sentido de respeto por las condiciones naturales de cada espacio territorial, si se quiere, en realidad, construir pilares para soportar escenarios prospectivos de desarrollo armónico.

Según el DNP (2013a) esta integración constituye el escenario que permite la articulación de acciones sectoriales, públicas y privadas, de forma transversal, con el fin de estructurar proyectos integrales de desarrollo regional, con visión a largo plazo, que demandarán la existencia de acuerdos entre el gobierno Nacional y entidades territoriales, como esquemas asociativos, pero al unir esfuerzos de tipo supramunicipal, no se está realizando, en vía directa, una acción regional, asunto que se tiende a confundir, debido a las expectativas que se generan sobre la regionalización y que siguen sin resolverse, en el proceso de ordenamiento territorial (Posada \& Berrocal, 2015). Máxime cuando en la República de Colombia (2011), la Ley orgánica de Ordenamiento Territorial, ha creado una serie de figuras administrativas, para abrir la posibilidad de que los entes territoriales se unan para lograr resultados de desarrollo que, en realidad, han traído más confusión que oportunidades reales.

Una de tales oportunidades de asociatividad en Colombia, inducida más por la existencia de recursos financieros que por la intención originaria desde las localidades para trabajar en conjunto, es la que se impulsa por medio del instrumento Contrato Plan, específicamente, en los lineamientos de política 2014-2018 que, según el CONPES (2014), permite enlazar la ejecución de los proyectos al sistema de regalías, como parte del presupuesto púbico. Lo anterior, motiva analizar la extraña situación de Colombia: basta con remitirse a las estadísticas del DANE (2007), donde, en 1953, existían 817 municipios, y ya en el 2007, habían 1.100 municipios, es decir, en cinco décadas, aumentó el número en 283 municipios, lo que también se puede expresar como una atomización o separación del espacio, debido a intereses de tipo político administrativo, que no demuestra para nada asociatividad, por el contrario, ratifica el continuo proceso de disociación territorial, con lo cual, se resalta, en este artículo, la necesidad de unir esfuerzos, a través de acciones estratégicas, desde el seno de cada ente municipal y en conjunto con sus vecinos, para evitar mayores divisiones y aprovechar los elementos comunes de tipo geográfico-ambiental, que les permiten funcionar como un todo.

En lo que concierne a las estrategias de asociatividad, Fabre (2006) establece que los territorios se asocian para limitar su dependencia del gobierno central, preservando su autonomía en el acceso de recursos que le son asignados y plantea, dos tipos de estrategias utilizadas por las asociaciones de entidades territoriales, para potencializar los intercambios de sus fuerzas existentes: La creación de una situación de dependencia mutua, de manera que se estimule el poder de influencia entre sí y, la formalización de las relaciones y de los intercambios, que conlleve a un futuro más estable. En ambas, la proximidad geográfica facilita la relación entre las entidades asociadas y multiplica las oportunidades de encuentro. Para Donzelot (2003), el desarrollo de una estrategia de asociatividad consiste en aumentar la concentración de flujos de intercambio entre los municipios pertenecientes a la asociación, mientras que para Bally (2017) es la construcción de una comunicación intercomunal pertinente y centrada en el interés general de los asociados, ya que permite agrupar las problemáticas y las soluciones comunes y unas obligaciones colectivas capaces de co-producir desarrollo.

El referente internacional de la asociatividad es Francia, en donde, según Loubet (2013), la eficiencia de este país, en cuanto a cooperación local e intermunicipal, se traduce en recomposiciones territoriales y gubernamentales, debido a que los representantes de los municipios se confrontan, en el día a día, al ejercicio complejo de la cooperación y la definición de proyectos territoriales. En particular, una experiencia francesa de asociatividad, que sirve de referente para Casanare, es la del sindicato, conformado por los municipios de Arcueil, Cachan, Fresnes y L'hay-les -Roses, que se agruparon en el marco de una convención de asociación, para elaborar, conjuntamente y en cooperación con el Estado, un estatuto intermunicipal para el ambiente, lo que les permitió determinar objetivos para cada dominio ambiental, ya fuera aire, agua, desechos, energía o ruido y al examinar la transversalidad de las problemáticas, crearon una estrategia y un programa de acción al interior de un marco ambiental institucional común (Holec, 1998).

Por su parte, en un análisis sobre la asociatividad territorial europea, abordado por Pavani \& Estupiñán (2016), reseñan el término como un principio que está en relación con las fusiones y la cooperación local, de tipo estructural y funcional y concluyen que, en Colombia, aún corresponde a una figura de estrategia sin fuerza, para la organización territorial. En otros países, el concepto término, principio o elemento de asociatividad, no es muy utilizado. En Chile, por ejemplo, se emplea el término asociativismo, para referirse a acciones de gestión micro y macro institucional y asumen el asociativismo territorial, como una modalidad de agrupación de municipalidades, en torno a un proyecto común (Erazo Jiménez et al. 2012).

La APC (2017), en su balance sobre la asociatividad, desde la óptica internacional en Colombia, ha destacado que corresponde a una estrategia para la construcción de capital social y la generación de economías de escala, porque genera inclusión productiva e integración en cadenas productivas regionales y nacionales y porque funciona como modelo de cooperación entre actores, para el desarrollo territorial. Es, en esta vía, que el trabajo asociativo en el departamento del 
Casanare se hace importante, debido a que, por su potencial estratégico, ha pasado por situaciones de conflicto armado, que han disminuido los indicadores de derechos humanos y que, con urgencia, requiere nuevas formas de accionar, para reconstruir la confianza desde lo local (ONU, 2007). Específicamente en Casanare, existen antecedentes sobre asociatividad supramunicipal, como el realizado por la Asociación de Municipios del Ariari, quienes se han organizado buscando objetivos de desarrollo social (AMA, 2017). Este tipo de asociación es analizada por Ayala (2005), quien concluye, que corresponden a una forma de expresión local particular y que es una forma de manifestar la capacidad funcional de los territorios.

Parte de la problemática de Casanare es que está pasando por una transición en la que sus posibilidades de desarrollo se han visto menguadas, como consecuencia de la dependencia de los recursos provenientes de las regalías del petróleo y, por ello, necesita con urgencia explorar otras alternativas de desarrollo, que estén en estrecha relación con su potencial socioambiental, pero no extractivo. Al respecto, Vargas \& León (2016) identifican los efectos ambientales de la explotación petrolera en este lugar de Colombia, encontrando fuertes cambios en la cobertura boscosa y en los suelos agrícolas y, por ende, afectaciones a los ecosistemas y a las comunidades locales, que demuestran cómo, ni los municipios, ni el departamento, ni el país, han estado preparados para establecer modelos que consideren el potencial socio-ambiental, por encima de proyectos cortoplacistas de tipo extractivo.

A partir de lo anterior y para abordar el tema de este artículo, los autores proponen diferenciar tres tipos o tendencias de la asociativad así:

Asociatividad funcional: Corresponde a la ejecución de acciones jalonadas por un objetivo central, que genera una fuerza especial, con flujos que van desde y hacia adentro del lugar, que es el líder en la gestión para la ejecución de tales acciones, entre unidades que tienen elementos y dinámicas comunes. En términos de espacio, abarca los lugares con factores comunes de tipo geográfico-ambiental y, en términos de tiempo, se extiende gradualmente al largo plazo, porque al involucrar variables ambientales, el resultado es un proceso continuo, que se va consolidando en el corto, mediano y largo plazo.

Asociatividad estructural: Corresponde a una reorganización espacial y político-administrativa del gobierno local, que implica un nuevo mapa del territorio y una nueva forma de administración pública más compleja. En términos de espacio, se refiere al cambio que se le imprime a la división político-administrativa de algún ente territorial o a la sumatoria de divisiones político-administrativas precedentes y, en términos de tiempo, se concreta en la relación directa con los periodos de gobiernos de los entes involucrados, es decir, actualmente en Colombia, es de 4 años.

Asociatividad coyuntural: Corresponde a la aplicación de instrumentos, como planes, programas o proyectos puntuales, que obedecen a un objetivo o alcance puntual y a unos recursos financieros en correspondencia que, especialmente, están ligados al presupuesto destinado para la ejecución particular de las metas preestablecidas en cada instrumento plan, en un momento dado que, usualmente, se invierte, al acabarse el proyecto. En términos de espacio, se refiere, exclusivamente, al área de influencia del instrumento a ejecutar $\mathrm{y}$, en términos de tiempo, se determina por las metas a lograr, en especial, ligadas a la disponibilidad financiera y, por lo regular, son de un año de duración.

En consecuencia, dado que la asociatividad es una acción estratégica, en este artículo, no se exalta la configuración de nuevas regiones, sino, en esencia, se destaca, como criterio para la integración, la posibilidad de que funcionen articuladamente las propuestas de tipo supramunicipal, como acciones, no solo públicas sino privadas, sectoriales y, especialmente, poblacionales. Expresado de manera figurada, si la naturaleza ha estado de acuerdo en trabajar como un sistema articulado, le es más fácil a los seres racionales propender por un desarrollo armónico, a partir de su ejemplo. Es por ello, que el objetivo de este escrito consiste en aplicar un método de análisis geográfico ambiental, que arroje resultados espacializables, en el marco de la gestión territorial, para aportar bases al departamento del Casanare, Colombia, conducentes a la práctica de estrategias colectivas supramunicipales.

\section{MATERIALES Y MÉTODO}

El sujeto de estudio geográfico o lugar de aplicación del objetivo fue el Departamento de Casanare, ubicado al Oriente de Colombia, en la región de la Orinoquia; limita con 5 departamentos y lo conforman 19 municipios, de los cuales, Yopal, es la capital. El estudio, se realizó en 4 etapas consecutivas a saber: en la primera, se identificaron los aspectos del subsistema ambiental y del socioeconómico, para establecer elementos espacializables de asociatividad, como parte del sistema territorial. La información de base, se obtuvo con las alcaldías municipales y con los datos de geoservicios disponibles en el país.

En la segunda etapa, se procedió a elaborar las figuras cartográficas, para visualizar el contenido de 12 variables: 6, correspondientes al subsistema ambiental y 6, al subsistema socioeconómico. Estos mapas fueron realizados con el sistema de información geográfica "ArcMap 10.3", a partir de diferentes fuentes de información cartográfica, trabaja- 
da en formato raster y vector. En la tercera etapa, se logró un acercamiento directo con la comunidad, aplicando una encuesta social de percepción aleatoria. Se logró recabar información con 10 preguntas, en cada uno de los 19 municipios, a un promedio de 10 personas, por cada uno, para un total de 190 entrevistas; realizar las encuestas fue fundamental para poder entender la situación de los municipios. Adicionalmente, en esta etapa, se revisó la existencia y el funcionamiento formal de las diferentes secretarías, encargadas oficialmente de los principales temas que representan el desarrollo local: educación, salud, desarrollo económico, planeación, hacienda, seguridad y convivencia, desarrollo social, infraestructura y obras públicas, ambiente y agricultura. El contar con estos resultados aportó a la investigación una mayor precisión, para dar cumplimento al objetivo trazado, ya que permite discretizar la percepción de la población sobre el potencial de asociatividad, desde los elementos geográfico-ambientales del territorio.

En la cuarta y última etapa, se diseñó la propuesta estratégica de asociatividad territorial, en aras del desarrollo para el departamento del Casanare y de sus municipios, para lo cual, se realizó un análisis y cruce de las variables, tanto del susbsistema ambiental como del socioeconómico, de manera que se pudiera determinar el grado homogeneidad, en diferentes entes territoriales, lo que derivó, en el planteamiento de una propuesta preliminar, que responde a necesidades y oportunidades compartidas entre varios municipios.

\section{RESULTADOS Y DISCUSIÓN}

De acuerdo con la metodología aplicada, se presenta, primero, la síntesis cartográfica de las variables consideradas, para obtener elementos comunes de tipo geográfico-ambiental, enlazado, no solo como un subsistema ambiental sino como subsistema socioeconómico y que, en esencia, suman el potencial para la funcionalidad entre entes territoriales locales, para una estrategia de asociatividad. Posteriormente, se presentan los resultados de las entrevistas a la comunidad, en relación con el tema y, para finalizar, la propuesta de asociatividad resultante.

La potencialidad del departamento de Casanare desde el susbsistema ambiental (Figura 1)

- Geología: predomina el lomerío, piedemonte y altiplanicie; constituye una zona de transición entre la llanura y la cordillera oriental, entre los 300 y 1.000 m.s.n.m., lo que potencia explotación de yacimientos de petróleo y gas.

- Recurso hídrico: se destacan los niveles de captación y de utilización de agua para actividades agrícolas y minero energéticas.

- Zonas de protección ambiental: se resaltan zonas de reserva forestal, zonas de alta biodiversidad, ecosistemas frágiles y estratégicos, cuencas, páramos y humedales.

- Ecosistemas: predominan los biomas de tipo pedioboma y heliobioma, los pediobimas y corresponden a selvas de galería, que atraviesan áreas de sabana, con largos periodos de inundación.

- Erosión: se presenta erosión severa, causada por acciones antrópicas.

- Cobertura vegetal: el bosque tropical se encuentra, principalmente, en las montañas, piedemonte y en las orillas de los caños y de los ríos.

La potencialidad del departamento de Casanare desde el susbsistema social (Figura 2)

- Densidad poblacional: es baja y se considera una desventaja para lograr mayor cobertura de los servicios básicos, por asuntos de presupuesto.

- Resguardos indígenas: aportan un valor cultural incalculable, por el potencial de intercambio de conocimiento que se obtiene en estas comunidades; existen tres áreas, donde se localizan comunidades indígenas: el resguardo de Barronegro y Chaparral, Orocué y el resguardo de Mochuelo.

- Unidades industriales; solo existen 11 municipios con un rango bajo de industrias, lo que expresa una baja dinámica de desarrollo económico.

Consideraciones de la comunidad sobre el potencial de asociatividad territorial (Figura 3)

Se destaca que el $100 \%$ de los encuestados admiten que el Casanare es un buen lugar para vivir, lo que, de inmediato, lleva a pensar en la posibilidad de continuar construyendo un mejor futuro. La población de los municipios de Hato Corozal, Paz de Ariporo, Trinidad, San Luis de Palenque, Orocue, Villanueva y Tauramena le dan mucha importancia al medio natural, especialmente, a la belleza paisajística que ofrece este territorio, asunto que potencia la asociatividad, con actividades propuestas por la población en función del ecoturismo. El poblador de Casanare ve, como positivo, la posibilidad de asociarse entre municipios, principalmente, para mantener sus tradiciones, como la ganadería y para innovar en actividades agroindustriales.

\section{Capacidad administrativa local para el desarrollo en los} entes territoriales de Casanare (Cuadro 1)

Sin excepción, tanto la administración de los 19 municipios como la de la gobernación, cuentan con una secretaría general de gobierno. La diferenciación, se presenta en los otros diez temas relativos al desarrollo local, que como se puede apreciar en los datos obtenidos, demuestran una fuerte contradicción entre el potencial del subsistema ambiental y la disponibilidad de recursos para su administración y manejo, principalmente, en lo relativo a la secretaría de ambiente y a la de agricultura y aunque la mayoría cuenta con secretaría 


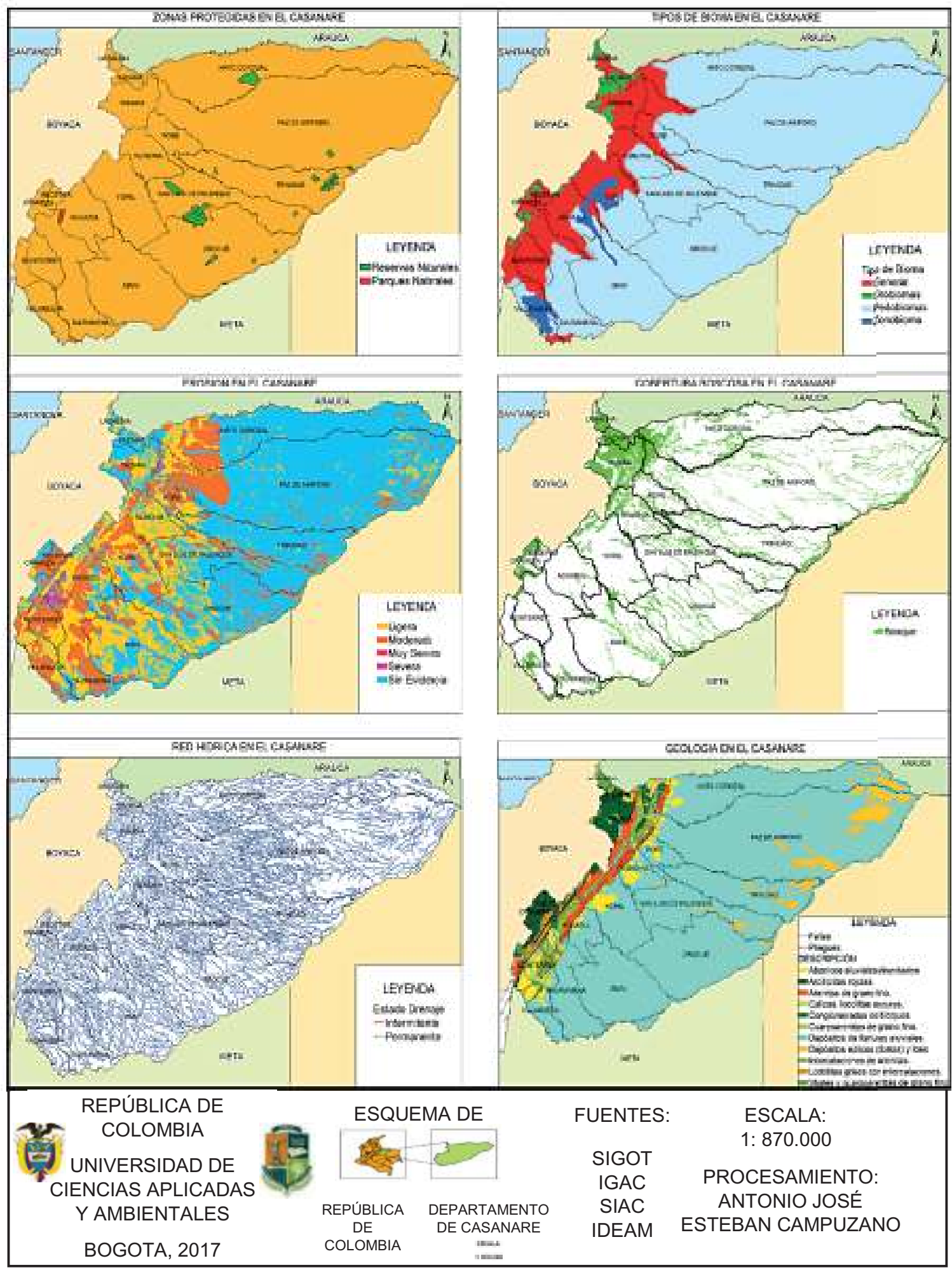

Figura 1. La potencialidad del departamento de Casanare desde el susbsistema ambiental. 

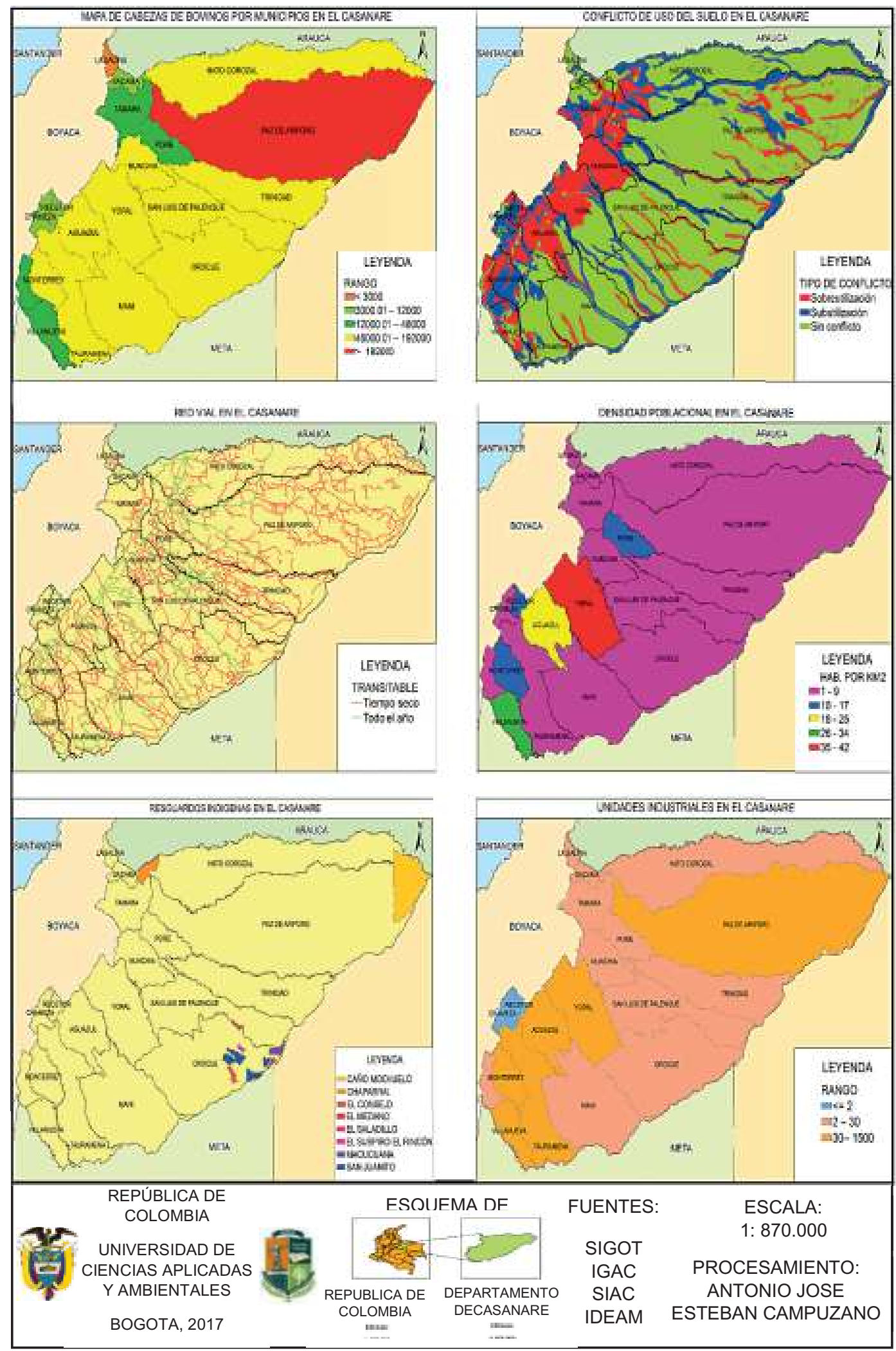

Figura 2. La potencialidad del departamento de Casanare desde el susbsistema socioeconomico. 
1. ¿Considera que El Casanare es un buen lugar para vivir?

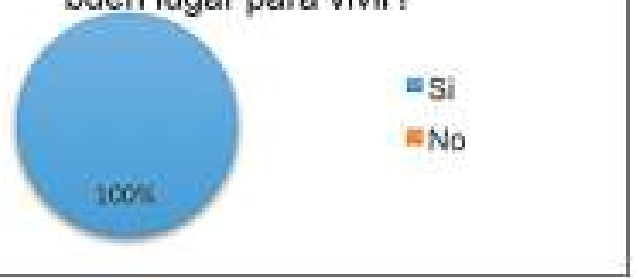

3. ¿Qué tipo de actividades considera posible realizarlas en conjunto con

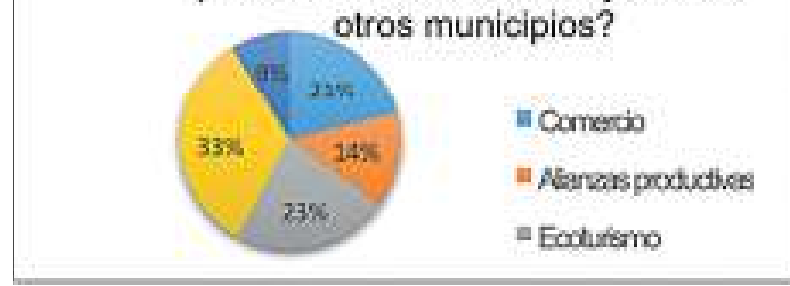
tros municipios?

5. ¿Cómo le gustaría ver a los municipios en 20 años?

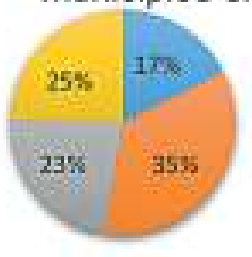

ETustion

- Genadero

= Agricole

industral
7. ¿Conoce acerca sobre las

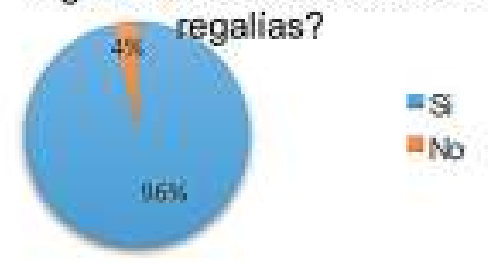

\section{9. ¿Qué proyectos estan teniendo} más repercusión actualmente?

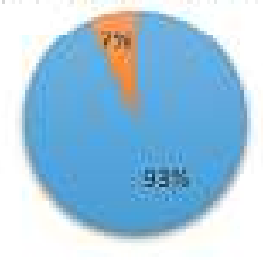

2. ¿Qué aspectos geográfico ambientales destaca de Casanare?

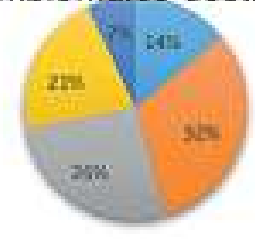

4. ¿Dificultades para trabajar de manera asociada con los municipios del departamento?

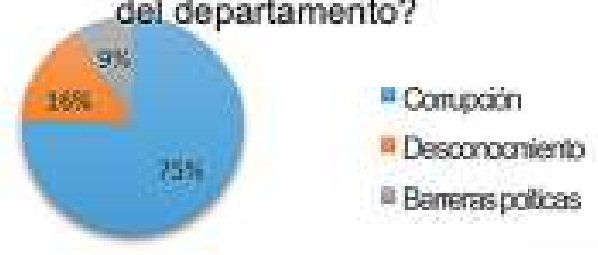

\section{6. ¿Conoce los instrumentos de} asociatividad territorial?

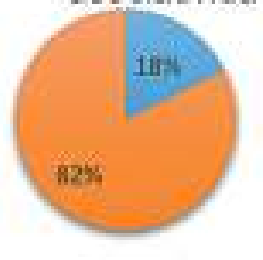

ESi

ENo
8. ¿Qué proyectos sugiere para dare mejor manejo a las regalias?

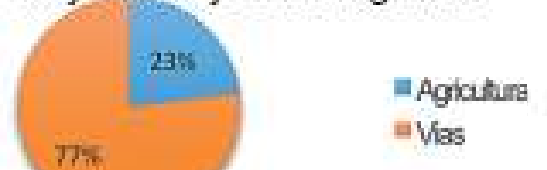

10. ¿Siente que la economía local cada dia es más compleja?

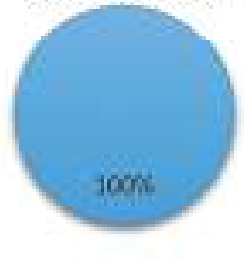

as

a $\mathrm{No}$

Figura 3. Consideraciones de la comunidad sobre el potencial de asociatividad territorial. 
Cuadro 1. Capacidad administrativa local para el desarrollo, en los entes territoriales de Casanare.

\begin{tabular}{|c|c|c|c|c|c|c|c|c|c|c|}
\hline ENTE TERRITORIAL & $\begin{array}{l}\text { S. EDU- } \\
\text { CACIÓN }\end{array}$ & $\begin{array}{c}\text { S. } \\
\text { SALUD }\end{array}$ & $\begin{array}{l}\text { S. DESARRO- } \\
\text { LLO ECONÓ- } \\
\text { MICO }\end{array}$ & $\begin{array}{l}\text { S. } \\
\text { PLANEA- } \\
\text { CIÓN }\end{array}$ & $\begin{array}{l}\text { S. } \\
\text { HA- } \\
\text { CIEN- } \\
\text { DA }\end{array}$ & $\begin{array}{c}\text { S. } \\
\text { SEGU- } \\
\text { RIDAD Y } \\
\text { CONVI- } \\
\text { VENCIA }\end{array}$ & $\begin{array}{c}\text { S. } \\
\text { DESA- } \\
\text { RROLLO } \\
\text { SOCIAL }\end{array}$ & $\begin{array}{c}\text { S. IN- } \\
\text { FRAES } \\
\text { Y OBRAS } \\
\text { PUBLI- } \\
\text { CAS }\end{array}$ & $\begin{array}{c}\text { S. } \\
\text { AM- } \\
\text { BIENTE }\end{array}$ & $\begin{array}{c}\text { S. } \\
\text { AGRICUL- } \\
\text { TURA }\end{array}$ \\
\hline CASANARE & $\mathrm{X}$ & $\mathrm{X}$ & & $\mathrm{X}$ & $\mathrm{X}$ & & $\mathbf{X}$ & $\mathrm{X}$ & $\mathrm{X}$ & $\mathrm{X}$ \\
\hline YOPAL & $\mathrm{X}$ & $\mathrm{X}$ & $\mathrm{X}$ & & $\mathrm{X}$ & & & & & \\
\hline AGUAZUL & $\mathrm{X}$ & & $\mathrm{X}$ & & $\mathrm{X}$ & & & $\mathrm{X}$ & $\mathrm{X}$ & \\
\hline CHÁMEZA & & & & $\mathrm{X}$ & $\mathrm{X}$ & & $\mathrm{X}$ & $\mathrm{X}$ & & \\
\hline HATO COROZAL & & & & $\mathrm{X}$ & $\mathrm{X}$ & & & & & \\
\hline LA SALINA & & & & $\mathrm{X}$ & $\mathrm{X}$ & & & $\mathrm{X}$ & & \\
\hline MANÍ & & & & & $\mathrm{X}$ & & $X$ & $\mathrm{X}$ & $X$ & \\
\hline MONTERREY & & & $\mathrm{X}$ & & $\mathrm{X}$ & $\mathrm{X}$ & $\mathrm{X}$ & $\mathrm{X}$ & $\mathrm{X}$ & \\
\hline NUNCHÍA & & & & $\mathrm{X}$ & $\mathrm{X}$ & & $\mathrm{X}$ & $\mathrm{X}$ & & \\
\hline OROCUÉ & & & & $\mathrm{X}$ & $\mathrm{X}$ & & $\mathrm{X}$ & $\mathrm{X}$ & & \\
\hline PAZ ARIPORO & & $X$ & & $\mathrm{X}$ & & & & $\mathrm{X}$ & & $X$ \\
\hline PORE & & & $\mathrm{X}$ & $\mathrm{X}$ & $X$ & & $\mathrm{X}$ & $\mathrm{X}$ & & \\
\hline RECETOR & & & & $\mathrm{X}$ & & & $\mathrm{X}$ & $\mathrm{X}$ & & \\
\hline SABANALARGA & & & & $\mathrm{X}$ & $\mathrm{X}$ & & $\mathrm{X}$ & $\mathrm{X}$ & & \\
\hline SÁCAMA & & & & $\mathrm{X}$ & & & & & & \\
\hline SAN LUIS PALENQUE & & & & $\mathrm{X}$ & $\mathrm{X}$ & & $\mathrm{X}$ & $\mathrm{X}$ & & \\
\hline TÁMARA & & & $\mathrm{X}$ & $\mathrm{X}$ & $\mathrm{X}$ & & $X$ & $\mathrm{X}$ & $\mathrm{X}$ & \\
\hline TAURAMENA & & & $\mathrm{X}$ & & $\mathrm{X}$ & & $\mathrm{X}$ & $\mathrm{X}$ & & \\
\hline TRINIDAD & $X$ & $\mathrm{X}$ & & & $\mathbf{X}$ & & & $\mathrm{X}$ & & \\
\hline VILLANUEVA & & & $\mathrm{X}$ & $\mathrm{X}$ & $\mathrm{X}$ & & $\mathrm{X}$ & $\mathrm{X}$ & $\mathrm{X}$ & \\
\hline
\end{tabular}


de planeación y de hacienda, lo que se esperaría es que hubiera una propuesta de manejo administrativo más acorde con las potencialidades del Departamento.

\section{Propuesta estratégica de asociatividad territorial (Figura 4)}

Para la estrategia de gestión territorial, se seleccionaron seis municipios entre 19, con los cuales, se puede llevar a cabo una asociatividad preliminar en el Departamento de Casanare, que jalone, posteriormente, otros procesos: Hato Corozal, Paz de Ariporo, Pore Trinidad, San Luis de Palenque y Orocue. La elección, se hizo, por una parte, debido a sus potencialidades ambientales conjuntas y, por otra, por el potencial de las variables sociales y las respuestas de la comunidad. Se destaca el potencial hídrico que, a su vez, puede ser utilizado como fuentes de transporte; se ubica el mayor número de reservas indígenas, que representan la multiculturalidad; tienen una economía estable con vocación agrícola y ganadera y, actualmente, en menor proporción, minero-energética; son, además, los entes territoriales que, según la comunidad, motivan el ejemplo para construir un mejor futuro en conjunto. Las acciones para desarrollar la estrategia de asociatividad territorial deberán estar enlazadas por actividades de: agroindustria amigable con el ambiente, ecoturismo, conectividad (vial, eléctrica, de comunicación), protección y manejo de los ecosistemas estratégicos y de la diversidad étnica y cultural. Para gestionar los recursos compartidos es fundamental mantener estas acciones como prioritarias y darles continuidad, en el largo plazo y, en ningún momento, como una sumatoria de proyectos cortoplacistas, lo cual, permitirá un desarrollo más homogéneo y sostenido.

Con los resultados logrados, se evidenció, no solo la necesidad de hacer más visible al Departamento del Casanare, en Colombia, sino la dificultad de aplicar una metodología que tenga en consideración el deseo de las comunidades locales, ya que el acceso a la zona y a la información son limitadas. Además, la diversidad territorial exige, por sí, una variedad de apuestas entre municipios socios, dependiendo de sus intereses. Una propuesta en una vía similar es la que realizan Cáceres et al. (2013), para el municipio de Yopal, en la que resaltan la oportunidad de organizarse entre pequeños y medianos productores agrícolas y la dinamización del turismo asociativo integrado a cadenas productivas agrícolas, para el desarrollo de la ruralidad.

Por otra parte, se ha observado una expectativa coyuntural y cortoplacista, propiciada por el Estado, en la intención de distribuir recursos, a nivel de región, pero que no es bien comprendida por las comunidades, a quienes les urge resolver una cantidad de demandas sociales acumuladas, a través de alguna oportunidad de recursos financieros, que pudiera surgir con la elaboración de un proyecto, lo cual, no excluye la opción de aprovechar la priorización de proyectos de in- versión, sean de la Nación, del Departamento, de recursos del Sistema General de Regalías -por parte de los Órganos Colegiados de Administración y Decisión-, de la Corporación Autónoma de los Recursos Naturales o de la sumatoria de recursos financieros locales, siempre y cuando se le dé continuidad a estas mismas acciones, en el mediano y largo plazo, para motivar a otros municipios a asociarse, de manera estratégica, hasta que se logre cobertura por subregiones, en todo el departamento.

Entonces, en localidades que, por largos periodos han buscado su desarrollo tan solo de manera endógena y no exógena, la opción de ver a su vecino, como parte de un todo, que posibilita un desarrollo conjunto y que le trae bienestar para ambos, se convierte en una oportunidad que, al final, se concreta en la gestión territorial. Se insiste en la diferencia sobre la asociatividad, como estrategia de desarrollo regional, que parte de una directriz nacional, DNP (2013b), porque aquí se resalta es la asociatividad, que nace de la gestión local y jalona procesos de desarrollo supramuniciapal, en un territorio con factores comunes, especialmente, de tipo geográfico-ambiental, lo que está en la misma vía de lo que plantean Moreno et al. (2014), quienes aconsejan que, para el futuro de Colombia, en el marco del postconflicto, se debe es incentivar la asociatividad municipal, con el fin de articular planes y fondos de inversión.

En este punto, es importante recordar el recorrido de Francia, en materia de asociatividad, en donde, actualmente, existen diversos tipos de asociaciones, según las capacidades que despliegan como gestores, para estructurar proyectos suficientemente atractivos, originales e innovadores y para conseguir recursos financieros, que les permitan hacer real los proyectos (Pasquier, 2012).

En conclusión, el Departamento, como unidad intermedia entre los municipios y la Nación, debe motivar, coordinar y articular procesos de largo plazo, de tipo supramunicipal, con motores en localidades claves, que promueva objetivos de desarrollo sostenible y que logren, en primera instancia, concretar la estrategia de asociatividad funcional. Si un municipio ve como una estrategia de desarrollo la oportunidad de asociarse es porque tiene la posibilidad de ofrecer algo positivo a su vecino o porque tienen una serie de necesidades que se puede resolver de manera compartida, ya que cuentan con ofertas naturales similares y demandas sociales conjuntas.

En Colombia existen varias posibilidades de hacer proyectos en conjunto, pero si corresponden solamente a una asociatividad coyuntural; esta posibilidad nace y muere con el proyecto puntual. mientras que los proyectos de tipo funcional, basados en el potencial socioambiental, le permiten duración, consolidación y multiplicación. La estrategia de asocia- 


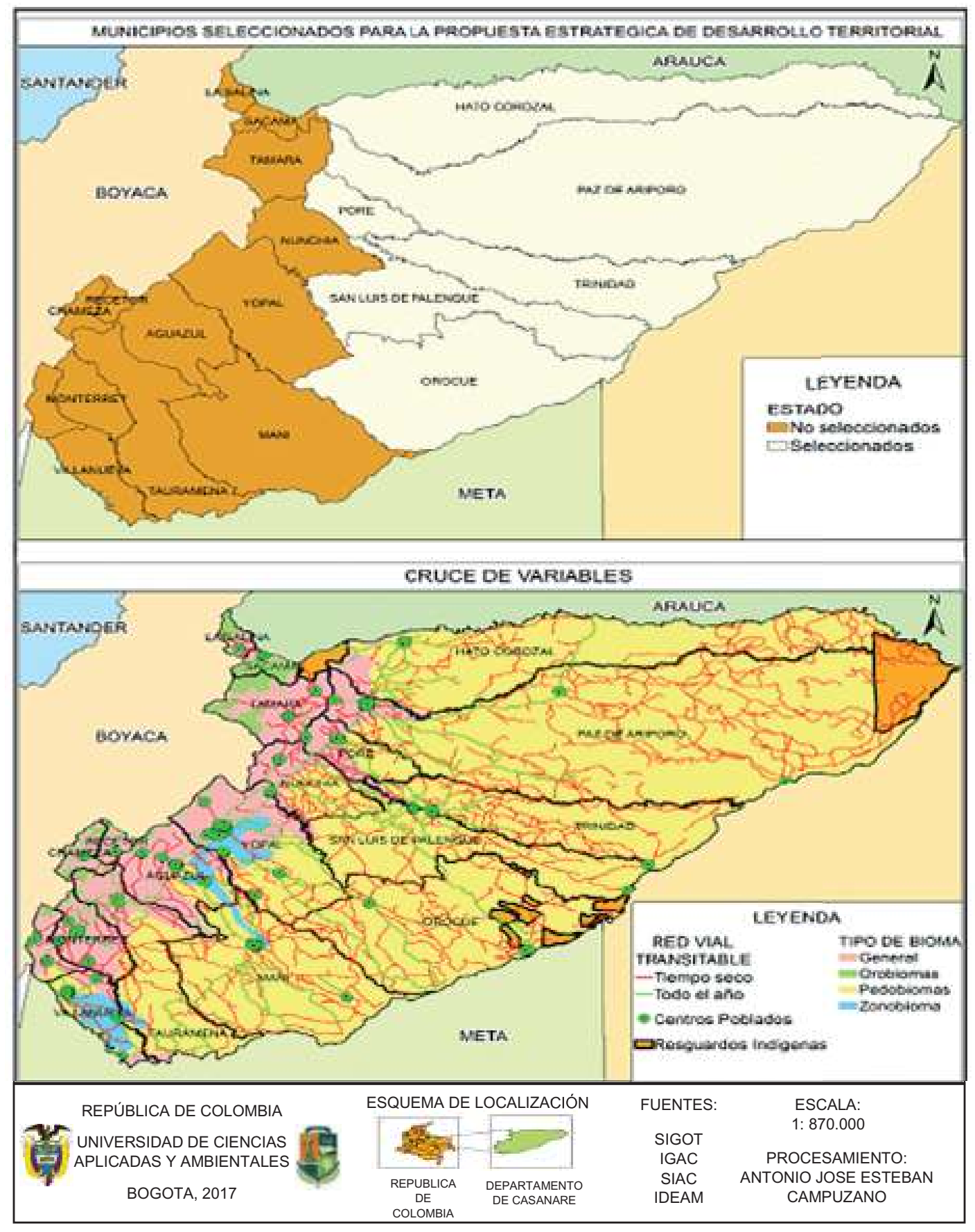

Figura 4. Propuesta estratégica de asociatividad territorial en el departamento del Casanare. 
tividad es el pegamento de los intereses comunes; lo natural es la base y lo social es lo que establece la ruptura o la unión; por ello, el ejercicio de asociatividad, se refleja en la capacidad de ARTICULACIÓN. La desarticulación es consecuencia de la falta de acción y de gestión local, pero con la asociatividad, se potencian las acciones que generan un mejor futuro. La visión de desarrollo supramunicipal debe partir desde la base, porque son los municipios los que deben establecer los factores comunes, que los remiten a dinamizar la estrategia de asociarse.

En las circunstancias de Colombia, se sugiere aprovechar la oportunidad de los instrumentos plan de ordenamiento departamental y de ajustar los planes de ordenamiento territorial municipal, de manera articulada, en donde el Departamento no pretenda sustituir al Municipio, como célula de planificación y gestión territorial, sino que lo apoye en procesos que trascienden en el tiempo, en el espacio y en los objetivos, como son las estrategias de asociatividad territorial, capaces de representar la concurrencia, a partir de un análisis de posibles proyectos de base natural y social, ya que estos proyectos son los que mueven los intereses reales y no los creados por coyunturas políticas. La asociatividad funcional, tratada en este escrito, quiere confirmar el mensaje de la naturaleza, sobre mantener unidos los elementos del subsistema ambiental y potenciarlos con las capacidades del subsistema social.

Agradecimiento: Los autores agradecen a la U.D.C.A, por financiar el proyecto de investigación "La coherencia de la gestión territorial en Colombia", que dio origen a este manuscrito. Conflicto de Intereses: El manuscrito fue preparado y revisado por los autores y declaran ser quienes originaron el artículo y no presentan conflicto de intereses.

\section{BIBLIOGRAFÍA}

1. AGENCIA PRESIDENCIAL DE COOPERACIÓN -APC-. 2017. Asociatividad. Balance de las experiencias de cooperación internacional en Colombia. 24p. Disponible desde Internet en: https:/www.apccolombia.gov.co/sites/default/files/archivos_usuario/ publicaciones/asociatividad-baja.pdf (con acceso 13/08/2017).

2. AYALA, M. 2005. Las asociaciones de municipios en Colombia. El marco normativo frente a la realidad institucional. Rev. Opera. 5(5):45-54.

3. ASOCIACIÓN DE MUNICIPIOS DEL ARIARI -AMA-. 2017. Disponible desde Internet en: http://web.archive.org/web/20130816061828/http://www.a-m-a. com.co/ (con acceso 21/04/017).
4. BALLY, J.L. 2017. Communication, Guides des élus. Un ouvrage juillet. DIRCOM 10. Francia.

5. CABEZA, I. 2015. Cohesión territorial: organización para reducir los desequilibrios territoriales. Bitácora. 25(1):51-56.

6. CÁCERES G., L.S.; PARDO E., C.E.; TORRES, A.M. 2013. La asociatividad y las cadenas productivas: una alternativa de desarrollo para el municipio de Yopal, Casanare. Cooperativismo \& Desarrollo, 21(102):69-75.

7. CONSEJO NACIONAL DE POLÍTICA ECONÓMICA Y SOCIAL -CONPES-. 2014. República de Colombia. Departamento Nacional de Planeación. Documento 3822, 40p.

8. DEPARTAMENTO NACIONAL DE ESTADÍSTICA DANE-. 2007. Documento DIVIPOLA. Disponible desde Internet en https://www.dane.gov.co/files/ investigaciones/divipola/divipola.pdf (con acceso 17/06/2017).

9. DEPARTAMENTO NACIONAL DE PLANEACIÓN -DNP-. 2013a. Comité Especial Interinstitucional. Definición legal y funcional de los esquemas asociativos en Colombia. 81p.

10. DEPARTAMENTO NACIONAL DE PLANEACIÓN -DNP-. 2013b. Balance preliminar de los procesos asociativos en Colombia. Departamento Nacional de Planeación. Colombia. 45p.

11. DONZELOT, J. 2003. Faire société: La Politique de la ville aux Etats-Unis et en France. La couleur des idées. $368 p$.

12. ERAZO JIMÉNEZ, M.S.; VILLALTA PÁUCAR, M.; MORALES JELDES, M. 2012. Representaciones sociales del asociativismo municipal en educación: Un marco conceptual para la educación chilena. Polis (Santiago). 11(31):141-156.

13. FABRE, P. 2006. Les associations face aux communes: quels leviers d'actions pour l'appropriation des ressources. Journée de Recherche «Quel management pour les associations?. CERMAT-LOG. 18p.

14. HOLEC, N. 1998. Création d'un syndicat intercommunal à Arcueil, Cachan, Fresnes, LHay-les-Roses- dialogues, propositions, histoires pour une citoyenneté mondiale 
15. LOUBET. 2013. Lapprentissage de la coopération intercommunale: modalités et instruments, Lespace politique. Revue en ligne, $\mathrm{n}^{\circ}$ 18. Disponible desde Internet en: http://espacepolitique.revues.org/2454 (con acceso 15/06/2017).

16. MORENO, C.; TRUJILLO, A.; VELÁSQUEZ, F.; RONDÓN C.; GARZÓN, C.; RESTREPO, D. 2014. Descentralización en clave de paz. Fundación Konrad Adenauer. Colombia. 106p.

17. ORGANIZACIÓN DE LAS NACIONES UINIDAS -ONU-. 2007. Derechos Humanos y DIH: Diagnóstico Departamental Casanare. 14p. Disponible desde Internet en: http://www.acnur.org/t3/uploads/media/ COI_2169.pdf (con acceso 20/09/2017).

18. PAVANI, G.; ESTUPIÑÁN A, L. 2016. Déficit municipal y compleja circulación de la figura de la asociatividad territorial: Lectura comparada para comprender el caso colombiano. Civilizar. 16(30):21-50.

19. PASQUIER, R. 2012. Le pouvoir régional. Mobilisations, décentralisation et gouvernance en France.Questions de communication 23. Presses de Sciences po, coll. 380p.
20. POSADA, A.; BERROCAL, A. 2015. Reconfiguración regional en el marco del ordenamiento territorial; Francia, un espejo para Colombia. Rev. U.D.C.A Act. \& Div. Cient. 18(1):271-281.

21. POSADA, A. 2014. De la planeación a la gestión ambiental colectiva: posibilidades en Bogotá - Colombia. Rev. U.D.C.A. Act. \& Div. Cient. 17(1):257-264.

22. REPÚBLICA DE COLOMBIA. 2011. Ministerio del interior y justicia. Ley orgánica de Ordenamiento Territorial, 1454, 34p.

23. ROJAS, J. 2016. Post-acuerdo y gestión territorial en Colombia. Bitácora 26(2):135-146.

24. VARGAS, Y.; LEÓN, N. 2016. Exploración y explotación de petróleo: enfoque geográfico de los efectos ambientales en el Piedemonte llanero. Perspectiva Geográfica. 21(2):199-224.

Recibido: Julio 18 de 2017

Aceptado: Sept. 29 de 2017

\section{Cómo citar:}

Posada Arrubla, A.; Campuzano, A.E.; Berrocal Mendoza, A.E. 2017. gestión territorial mediante estrategia de asociatividad para Casanare -Colombia. Rev. U.D.C.A Act. \& Div. Cient.20(2): 445-456. 